ANNALES

POLONICI MATHEMATICI

LXXVII.3 (2001)

\title{
The kernel theorem for Laplace ultradistributions
}

\author{
by SŁaWomir Michalik (Warszawa)
}

Abstract. A kernel theorem for spaces of Laplace ultradistributions supported by an $n$-dimensional cone of product type is stated and proved.

Introduction. Laurent Schwartz showed in [5] that for every continuous linear map $A: D(\Omega) \rightarrow D^{\prime}(\Omega)$ there exists a unique distribution $K \in D^{\prime}(\Omega \times \Omega)$, called the distributional kernel of the operator $A$, such that

$$
A[\varphi][\psi]=K[\varphi \otimes \psi] \quad \text { for } \varphi, \psi \in D(\Omega) .
$$

In this paper we give the kernel theorem for the space $L_{(\omega)}^{\prime\left(M_{p}\right)}(\Gamma)$ of Laplace ultradistributions supported by an $n$-dimensional cone $\Gamma$ of product type (i.e. $\left.\Gamma=v+\left(\overline{\mathbb{R}}_{+}\right)^{n}\right)$. Namely for any continuous linear map $A: L_{\left(\omega_{1}\right)}^{\left(M_{p}\right)}\left(\Gamma_{1}\right) \rightarrow L_{\left(\omega_{2}\right)}^{\prime\left(M_{p}\right)}\left(\Gamma_{2}\right)$ there exists $K \in L_{\left(\omega_{1}, \omega_{2}\right)}^{\prime\left(M_{p}\right)}\left(\Gamma_{1} \times \Gamma_{2}\right)$ such that (1) holds for all $\varphi \in L_{\left(\omega_{1}\right)}^{\left(M_{p}\right)}\left(\Gamma_{1}\right), \psi \in L_{\left(\omega_{2}\right)}^{\left(M_{p}\right)}\left(\Gamma_{2}\right)$. The proof of this theorem is based on the proof of the $S^{\prime}$-version of the kernel theorem given in [7].

Notation. We use the vector notation. In particular, if $a, b, v \in \mathbb{R}^{n}$ then $a<b$ means $a_{i}<b_{i}$ for $i=1, \ldots, n,[v, \infty)$ means $\left[v_{1}, \infty\right) \times \ldots \times\left[v_{n}, \infty\right)$ and $x^{z}$ means $x_{1}^{z_{1}} \ldots x_{n}^{z_{n}}$ for $x \in \mathbb{R}_{+}^{n}, z \in \mathbb{C}^{n}$.

Let $\Gamma \subseteq U \subseteq \mathbb{R}^{n}$ be such that $U$ is open in $\mathbb{R}^{n}, \Gamma$ is relatively closed in $U$ and $\Gamma \subseteq \overline{\operatorname{int} \Gamma}$ (i.e. $\Gamma$ is a fat set). Then for $k \in \mathbb{N}_{0} \cup\{\infty\}$,

$$
C^{k}(\Gamma):=\left\{f: \Gamma \rightarrow \mathbb{C}: \text { there exists } g \in C^{k}(U) \text { such that }\left.g\right|_{\Gamma}=f\right\} .
$$

We write $D$ for the differential operator $d / d x$.

Let $\left\{P_{\tau}\right\}_{\tau \in T}$ be a family of multinormed vector spaces. Then $\lim _{\tau \in T} P_{\tau}$ (resp. $\varliminf_{\tau \in T} P_{\tau}$ ) denotes the inductive limit (resp. projective limit) of $P_{\tau}$, $\tau \in T$.

2000 Mathematics Subject Classification: Primary 46F05.

Key words and phrases: Laplace ultradistributions, kernel theorem. 
Laplace ultradistributions. Let $\left(M_{p}\right)_{p \in \mathbb{N}_{0}}$ be a sequence of positive numbers satisfying the conditions (see [2]):

(M.0) $\quad M_{0}=M_{1}=1$.

(M.1) $\quad M_{p}^{2} \leq M_{p-1} M_{p+1}$ for $p \in \mathbb{N}$.

(M.2) There are constants $A, H$ such that

$$
M_{p} \leq A H^{p} \min _{0 \leq q \leq p} M_{q} M_{p-q} \quad \text { for } p \in \mathbb{N}_{0} .
$$

(M.3) There is a constant $A$ such that

$$
\sum_{q=p+1}^{\infty} \frac{M_{q-1}}{M_{q}} \leq A p \frac{M_{p}}{M_{p+1}} \quad \text { for } p \in \mathbb{N}_{0} .
$$

The associated function $M$ of the sequence $\left(M_{p}\right)$ is defined by

$$
M(\varrho):=\sup _{p \in \mathbb{N}_{0}} \log \frac{\varrho^{p}}{M_{p}} \quad \text { for } \varrho>0 .
$$

An ultradifferential operator $P(D)$ of class $\left(M_{p}\right)$ is defined by

$$
P(D):=\sum_{\alpha \in \mathbb{N}_{0}^{n}} a_{\alpha} D^{\alpha},
$$

where the $a_{\alpha} \in \mathbb{C}$ satisfy the condition: there are constants $K, C<\infty$ such that

$$
\left|a_{\alpha}\right| \leq C \frac{K^{|\alpha|}}{M_{|\alpha|}} \quad \text { for } \alpha \in \mathbb{N}_{0}^{n}
$$

The entire function $\mathbb{C}^{n} \ni z \mapsto P(z)$ is called a symbol of class $\left(M_{p}\right)$.

Definition 1 (see [3]). Let $v \in \mathbb{R}^{n}, \Gamma:=v+\left(\overline{\mathbb{R}}_{+}\right)^{n}=[v, \infty), \omega \in$ $(\mathbb{R} \cup\{\infty\})^{n}$. The space $L_{(\omega)}^{\prime\left(M_{p}\right)}(\Gamma)$ of Laplace ultradistributions is defined as the dual space of

$$
L_{(\omega)}^{\left(M_{p}\right)}(\Gamma):={\underset{a<\omega}{\longrightarrow}}_{a<} L_{a}^{\left(M_{p}\right)}(\Gamma),
$$

where for any $a \in \mathbb{R}^{n}$,

$$
L_{a}^{\left(M_{p}\right)}(\Gamma):=\lim _{h>0} L_{a, h}^{\left(M_{p}\right)}(\Gamma),
$$

and for any $h>0$,

$$
L_{a, h}^{\left(M_{p}\right)}(\Gamma):=\left\{\varphi \in C^{\infty}(\Gamma): q_{a, h, \Gamma}^{\left(M_{p}\right)}(\varphi):=\sup _{y \in \Gamma} \sup _{\alpha \in \mathbb{N}_{0}^{n}} \frac{\left|e^{-a y} D^{\alpha} \varphi(y)\right|}{h^{|\alpha|} M_{|\alpha|}}<\infty\right\} .
$$

Fix $\varepsilon>0$. We will construct a linear continuous extension mapping

$$
E_{\varepsilon}: L_{a}^{\left(M_{p}\right)}(\Gamma) \rightarrow L_{a}^{\left(M_{p}\right)}(-\varepsilon+\Gamma)
$$

such that $\operatorname{supp}\left(E_{\varepsilon} \varphi\right) \subset-\varepsilon / 2+\Gamma$ for every $\varphi \in L_{a}^{\left(M_{p}\right)}(\Gamma)$. 
Without loss of generality we can assume that $\varepsilon<1$. For $k \in \mathbb{N}_{0}^{n}$ let

$$
U_{k}:=\left\{x \in \mathbb{R}^{n}:-\varepsilon<x_{i}-v_{i}-k_{i}<1+\varepsilon \text { for } i=1, \ldots, n\right\}
$$

be a covering of $\Gamma=v+\left(\overline{\mathbb{R}}_{+}\right)^{n}$. Let $\left\{\psi_{k}\right\}_{k \in \mathbb{N}_{0}^{n}}$ be a locally finite partition of unity (see Proposition 5.2 in [2]) subordinate to $\left\{U_{k}\right\}_{k \in \mathbb{N}_{0}^{n}}$ such that:

1) $\psi_{k} \in L_{0}^{\left(M_{p}\right)}(-\varepsilon+\Gamma)$;

2) the family $\left\{\psi_{k}\right\}_{k \in \mathbb{N}_{0}^{n}}$ is equibounded in $L_{0}^{\left(M_{p}\right)}(-\varepsilon+\Gamma)$;

3) $\operatorname{supp} \psi_{k} \subset U_{k}$;

4) $\sum \psi_{k}(x)=1$ on $\Gamma$.

Furthermore, let $\widetilde{E}_{\varepsilon, k}$ be a linear continuous extension operator for ultradifferentiable functions on the compact set $\bar{U}_{k} \cap \Gamma$ (see Theorem 3.1 in [4]):

$$
\widetilde{E}_{\varepsilon, k}: \mathcal{E}^{\left(M_{p}\right)}\left(\bar{U}_{k} \cap \Gamma\right) \rightarrow \mathcal{E}^{\left(M_{p}\right)}\left(\mathbb{R}^{n}\right),
$$

such that:

1) $\operatorname{supp}\left(\widetilde{E}_{\varepsilon, k} \psi\right) \subset(-\varepsilon / 2, \varepsilon / 2]^{n}+U_{k} \cap \Gamma$ for every $\psi \in \mathcal{E}^{\left(M_{p}\right)}\left(\bar{U}_{k} \cap \Gamma\right)$;

2) if $\psi \in \mathcal{E}^{\left(M_{p}\right)}\left(\bar{U}_{k} \cap \Gamma\right)$ and $\operatorname{supp} \psi \subset U_{k} \cap \Gamma$ then $\operatorname{supp}\left(\widetilde{E}_{\varepsilon, k} \psi\right) \cap \Gamma=$ $\operatorname{supp} \psi$.

Observe that for every $k \in \mathbb{N}_{0}^{n}$ there exists $j \in\{0, \ldots, n\}$ such that $\bar{U}_{k} \cap \Gamma$ is isometric to $[-\varepsilon, 1+\varepsilon]^{j} \times[0,1+\varepsilon]^{n-j}$. Hence we may assume that:

3) the family $\left\{\widetilde{E}_{\varepsilon, k}\right\}_{k \in \mathbb{N}_{0}^{n}}$ of operators is equicontinuous.

Now we define $E_{\varepsilon}$ by

$$
E_{\varepsilon}(\varphi):=\sum_{k \in \mathbb{N}_{0}^{n}} \widetilde{E}_{\varepsilon, k}\left(\psi_{k} \varphi\right) \quad \text { for } \varphi \in L_{a}^{\left(M_{p}\right)}(\Gamma) .
$$

By the properties of the functions $\left\{\psi_{k}\right\}_{k \in \mathbb{N}_{0}^{n}}$ and the mappings $\left\{\widetilde{E}_{\varepsilon, k}\right\}_{k \in \mathbb{N}_{0}^{n}}$, $E_{\varepsilon}$ is an extension operator and we may estimate pseudonorms of $E_{\varepsilon}(\varphi)$ by appropriate pseudonorms of $\varphi$. Therefore $E_{\varepsilon}$ is a continuous linear extension mapping.

Following the proof of Proposition 5.1 in [7] and using the mapping $E_{\varepsilon}$ we conclude that the space $L_{a}^{\left(M_{p}\right)}(\Gamma)$ is complete.

Let $v_{1} \in \mathbb{R}^{n_{1}}, v_{2} \in \mathbb{R}^{n_{2}}, \Gamma_{1}:=\left[v_{1}, \infty\right), \Gamma_{2}:=\left[v_{2}, \infty\right), \omega_{1} \in(\mathbb{R} \cup\{\infty\})^{n_{1}}$, $\omega_{2} \in(\mathbb{R} \cup\{\infty\})^{n_{2}}$. We denote by $L_{\left(\omega_{1}\right)}^{\prime\left(M_{p}\right)}\left(\Gamma_{1}, L_{\left(\omega_{2}\right)}^{\prime\left(M_{p}\right)}\left(\Gamma_{2}\right)\right)$ the space of Laplace ultradistributions on $\Gamma_{1}$ with values in $L_{\left(\omega_{2}\right)}^{\prime\left(M_{p}\right)}\left(\Gamma_{2}\right)$, i.e.

$$
A \in L_{\left(\omega_{1}\right)}^{\prime\left(M_{p}\right)}\left(\Gamma_{1}, L_{\left(\omega_{2}\right)}^{\prime\left(M_{p}\right)}\left(\Gamma_{2}\right)\right)
$$

if for any $\varphi \in L_{\left(\omega_{1}\right)}^{\left(M_{p}\right)}\left(\Gamma_{1}\right)$ we have $A[\varphi] \in L_{\left(\omega_{2}\right)}^{\prime\left(M_{p}\right)}\left(\Gamma_{2}\right)$ and the mapping

$$
L_{\left(\omega_{1}\right)}^{\left(M_{p}\right)}\left(\Gamma_{1}\right) \ni \varphi \mapsto A[\varphi] \in L_{\left(\omega_{2}\right)}^{\prime\left(M_{p}\right)}\left(\Gamma_{2}\right)
$$

is linear and continuous. 
We say that a sequence $\left(A_{\nu}\right)_{\nu \in \mathbb{N}}$, where $A_{\nu} \in L_{\left(\omega_{1}\right)}^{\left(M_{p}\right)}\left(\Gamma_{1}, L_{\left(\omega_{2}\right)}^{\left(M_{p}\right)}\left(\Gamma_{2}\right)\right)$, converges to zero in $L_{\left(\omega_{1}\right)}^{\prime\left(M_{p}\right)}\left(\Gamma_{1}, L_{\left(\omega_{2}\right)}^{\prime\left(M_{p}\right)}\left(\Gamma_{2}\right)\right)$ if

$$
\lim _{\nu \rightarrow \infty} A_{\nu}[\varphi][\psi]=0 \quad \text { for every } \varphi \in L_{\left(\omega_{1}\right)}^{\left(M_{p}\right)}\left(\Gamma_{1}\right), \psi \in L_{\left(\omega_{2}\right)}^{\left(M_{p}\right)}\left(\Gamma_{2}\right) .
$$

Analogously, we say that a sequence $\left(\widetilde{A}_{\nu}\right)_{\nu \in \mathbb{N}}$, where $\widetilde{A}_{\nu} \in L_{\left(\omega_{1}, \omega_{2}\right)}^{\prime\left(M_{p}\right)}\left(\Gamma_{1} \times \Gamma_{2}\right)$, converges to zero in $L_{\left(\omega_{1}, \omega_{2}\right)}^{\prime\left(M_{p}\right)}\left(\Gamma_{1} \times \Gamma_{2}\right)$ if

$$
\lim _{\nu \rightarrow \infty} \widetilde{A}_{\nu}[\Phi]=0 \quad \text { for every } \Phi \in L_{\left(\omega_{1}, \omega_{2}\right)}^{\left(M_{p}\right)}\left(\Gamma_{1} \times \Gamma_{2}\right) .
$$

\section{The kernel theorem}

Theorem 1 (The kernel theorem). The mapping

$$
\mathcal{I}_{M_{p}}: L_{\left(\omega_{1}, \omega_{2}\right)}^{\prime\left(M_{p}\right)}\left(\Gamma_{1} \times \Gamma_{2}\right) \rightarrow L_{\left(\omega_{1}\right)}^{\prime\left(M_{p}\right)}\left(\Gamma_{1}, L_{\left(\omega_{2}\right)}^{\prime\left(M_{p}\right)}\left(\Gamma_{2}\right)\right)
$$

such that for any $\widetilde{A} \in L_{\left(\omega_{1}, \omega_{2}\right)}^{\left(M_{p}\right)}\left(\Gamma_{1} \times \Gamma_{2}\right)$,

(2) $\mathcal{I}_{M_{p}}(\widetilde{A})[\varphi][\psi]:=\widetilde{A}[\varphi \otimes \psi] \quad$ for $\varphi \in L_{\left(\omega_{1}\right)}^{\left(M_{p}\right)}\left(\Gamma_{1}\right), \psi \in L_{\left(\omega_{2}\right)}^{\left(M_{p}\right)}\left(\Gamma_{2}\right)$,

is a linear topological isomorphism of the space $L_{\left(\omega_{1}, \omega_{2}\right)}^{\prime\left(M_{p}\right)}\left(\Gamma_{1} \times \Gamma_{2}\right)$ onto $L_{\left(\omega_{1}\right)}^{\prime\left(M_{p}\right)}\left(\Gamma_{1}, L_{\left(\omega_{2}\right)}^{\prime\left(M_{p}\right)}\left(\Gamma_{2}\right)\right)$.

The proof is based on the Mazur-Orlicz theorem on the separate continuity of 2-linear functionals.

Theorem 2 (Mazur-Orlicz; Theorem 4.7.1 of [1]). Let $E^{1}, E^{2}$ be multinormed complete vector spaces with the topologies given by non-decreasing sequences of pseudonorms $q_{k}^{j}(j=1,2 ; k=0,1, \ldots)$. Then each separately continuous bilinear form $\Phi: E^{1} \times E^{2} \rightarrow \mathbb{C}$ is continuous, i.e. there exist constants $C<\infty$ and $k \in \mathbb{N}_{0}$ such that

$$
\left|\Phi\left(\zeta_{1}, \zeta_{2}\right)\right| \leq C q_{k}^{1}\left(\zeta_{1}\right) q_{k}^{2}\left(\zeta_{2}\right) \quad \text { for } \zeta_{1} \in E^{1}, \zeta_{2} \in E^{2} .
$$

Furthermore, we have

Theorem 3 (see Theorem 1.3 in [8]). Let $E_{k}^{j}(j=1,2 ; k=0,1, \ldots)$ be a Banach space with norm $q_{k}^{j}$ such that $E_{k+1}^{j} \subseteq E_{k}^{j}$ and $q_{k}^{j}\left(\zeta_{j}\right) \leq q_{k+1}^{j}\left(\zeta_{j}\right)$ for $\zeta_{j} \in E_{k+1}^{j}$. Let $E^{j}:=\varliminf_{k \in \mathbb{N}_{0}} E_{k}^{j}$. Assume that $K_{k+1}^{j}:=\left\{\zeta_{j} \in E_{k+1}^{j}\right.$ : $\left.q_{k+1}^{j}\left(\zeta_{j}\right) \leq 1\right\}$ is precompact in $E_{k}^{j}$. Let $\Phi_{\nu}: E^{1} \times E^{2} \rightarrow \mathbb{C}(\nu=1,2, \ldots)$ be separately continuous bilinear forms converging to zero, i.e.

$$
\lim _{\nu \rightarrow \infty} \Phi_{\nu}\left(\zeta_{1}, \zeta_{2}\right)=0 \quad \text { for every } \zeta_{1} \in E^{1}, \zeta_{2} \in E^{2} .
$$

Then there exist $k \in \mathbb{N}_{0}$ and a sequence $\varepsilon_{\nu} \rightarrow 0_{+}$such that $\left|\Phi_{\nu}\left(\zeta_{1}, \zeta_{2}\right)\right| \leq \varepsilon_{\nu} q_{k+1}^{1}\left(\zeta_{1}\right) q_{k+1}^{2}\left(\zeta_{2}\right) \quad$ for $\zeta_{1} \in E_{k+1}^{1}, \zeta_{2} \in E_{k+1}^{2}, \quad \nu=1,2, \ldots$ 
It is easily seen that the spaces $E^{1}:=L_{a_{1}}^{\left(M_{p}\right)}\left(\Gamma_{1}\right)$ and $E^{2}:=L_{a_{2}}^{\left(M_{p}\right)}\left(\Gamma_{2}\right)$ satisfy the assumptions of Theorems 2 and 3 .

In the proof of the kernel theorem we shall use a lemma which generalizes a theorem on the change of order of integration. The lemma is analogous to Theorem 18.11 of [6], so we omit its proof.

Lemma 1. Let $g: \mathbb{R}^{n} \times \Gamma \rightarrow \mathbb{R}$, where $\Gamma:=[v, \infty), v \in \mathbb{R}^{n}$, and let $a \in \mathbb{R}^{n}, h>0$. Put $g_{s}(x):=g(s, x)$, where $s \in \mathbb{R}^{n}, x \in \Gamma$. Assume that $g$ satisfies:

1. For any $\alpha \in \mathbb{N}_{0}^{n}, D_{x}^{\alpha} g(s, x)$ is continuous on $\mathbb{R}^{n} \times \Gamma$.

2. For any $s \in \mathbb{R}^{n}, g_{s} \in L_{a, h}^{\left(M_{p}\right)}(\Gamma)$.

3. For any $s_{0} \in \mathbb{R}^{n}, \lim _{s \rightarrow s_{0}} g_{s}=g_{s_{0}}$ in $L_{a, h}^{\left(M_{p}\right)}(\Gamma)$.

Let $\gamma \in C_{0}^{0}\left(\mathbb{R}^{n}\right)$ and $u \in L_{a, h}^{\prime\left(M_{p}\right)}(\Gamma)$. Then

$$
\int_{\mathbb{R}^{n}} \gamma(s) u\left[g_{s}\right] d s=u\left[\int_{\mathbb{R}^{n}} \gamma(s) g_{s} d s\right] .
$$

Let $\gamma \in C^{0}\left(\mathbb{R}^{n}\right)$ be such that $|\gamma(s)| q_{a, h, \Gamma}^{\left(M_{p}\right)}\left(g_{s}\right)\left(1+\left|s_{1}\right|\right)^{2} \ldots\left(1+\left|s_{n}\right|\right)^{2}<C$. Choose a sequence of functions $\gamma_{\nu} \in C_{0}^{0}\left(\mathbb{R}^{n}\right)$ such that $\gamma_{\nu} \rightarrow \gamma$ in $C^{0}\left(\mathbb{R}^{n}\right)$, $\left|\gamma_{\nu}\right| \leq|\gamma|$ and pass to the limit in the already proved formula for $\gamma_{\nu} \in$ $C_{0}^{0}\left(\mathbb{R}^{n}\right)$. Then we have

LEMma 2. Under the conditions of Lemma 1, (4) holds for functions $\gamma \in C^{0}\left(\mathbb{R}^{n}\right)$ such that $|\gamma(s)| q_{a, h, \Gamma}^{\left(M_{p}\right)}\left(g_{s}\right)\left(1+\left|s_{1}\right|\right)^{2} \ldots\left(1+\left|s_{n}\right|\right)^{2}<C$ for $s \in \mathbb{R}^{n}$ with some $C<\infty$.

Proof of Theorem 1. We first observe that the transformation $\mathcal{I}_{M_{p}}$ is well defined. Indeed, let $\widetilde{A} \in L_{\left(\omega_{1}, \omega_{2}\right)}^{\prime\left(M_{2}\right)}\left(\Gamma_{1} \times \Gamma_{2}\right)$. Then for any $a_{j}<\omega_{j}(j=1,2)$ there exist $h>0$ and $c<\infty$ such that

$$
\begin{aligned}
& \left|\mathcal{I}_{M_{p}}(\widetilde{A})[\varphi][\psi]\right|=|\widetilde{A}[\varphi \otimes \psi]| \\
& \leq c \sup _{x_{1} \in \Gamma_{1}} \sup _{\alpha_{1} \in \mathbb{N}_{0}^{n_{1}}} \frac{\left|e^{-a_{1} x_{1}} D_{x_{1}}^{\alpha_{1}} \varphi\left(x_{1}\right)\right|}{h^{\left|\alpha_{1}\right|} M_{\left|\alpha_{1}\right|}} \sup _{x_{2} \in \Gamma_{2}} \sup _{\alpha_{2} \in \mathbb{N}_{0}^{n_{2}}} \frac{\left|e^{-a_{2} x_{2}} D_{x_{2}}^{\alpha_{2}} \psi\left(x_{2}\right)\right|}{h^{\left|\alpha_{2}\right|} M_{\left|\alpha_{2}\right|}}
\end{aligned}
$$

for $\varphi \in L_{a_{1}}^{\left(M_{p}\right)}\left(\Gamma_{1}\right)$ and $\psi \in L_{a_{2}}^{\left(M_{p}\right)}\left(\Gamma_{2}\right)$. Thus $\mathcal{I}_{M_{p}}(\widetilde{A}) \in L_{\left(\omega_{1}\right)}^{\prime\left(M_{p}\right)}\left(\Gamma_{1}, L_{\left(\omega_{2}\right)}^{\prime\left(M_{p}\right)}\left(\Gamma_{2}\right)\right)$. If we have a sequence $\left(\widetilde{A}_{\nu}\right)_{\nu \in \mathbb{N}}$ convergent to zero in $L_{\left(\omega_{1}, \omega_{2}\right)}^{\prime\left(M_{p}\right)}\left(\Gamma_{1} \times \Gamma_{2}\right)$ then the sequence of the corresponding numbers $c_{\nu}$ in (5) is also convergent to zero and consequently the sequence $\left(\mathcal{I}_{M_{p}}\left(\widetilde{A}_{\nu}\right)\right)_{\nu \in \mathbb{N}}$ is convergent to zero in $L_{\left(\omega_{1}\right)}^{\prime\left(M_{p}\right)}\left(\Gamma_{1}, L_{\left(\omega_{2}\right)}^{\prime\left(M_{p}\right)}\left(\Gamma_{2}\right)\right)$. Thus the operator $\mathcal{I}_{M_{p}}$ is continuous. 
Now we construct a continuous inverse transformation

$$
\mathcal{I}_{M_{p}}^{-1}: L_{\left(\omega_{1}\right)}^{\prime\left(M_{p}\right)}\left(\Gamma_{1}, L_{\left(\omega_{2}\right)}^{\prime\left(M_{p}\right)}\left(\Gamma_{2}\right)\right) \rightarrow L_{\left(\omega_{1}, \omega_{2}\right)}^{\prime\left(M_{p}\right)}\left(\Gamma_{1} \times \Gamma_{2}\right)
$$

such that $\mathcal{I}_{M_{p}} \mathcal{I}_{M_{p}}^{-1}=\operatorname{Id}$ and $\mathcal{I}_{M_{p}}^{-1} \mathcal{I}_{M_{p}}=$ Id.

Fix $A \in L_{\left(\omega_{1}\right)}^{\prime\left(M_{p}\right)}\left(\Gamma_{1}, L_{\left(\omega_{2}\right)}^{\prime\left(M_{p}\right)}\left(\Gamma_{2}\right)\right)$ and take any $a_{1}, a_{2}, d_{1}, d_{2}$ such that $a_{j}<d_{j}<\omega_{j}(j=1,2)$. By Theorem 2 there exist $c_{A}<\infty, h>0$ such that

$$
\begin{aligned}
|A[\varphi][\psi]| \leq c_{A} q_{d_{1}, h, \Gamma_{1}}^{\left(M_{p}\right)}(\varphi) q_{d_{2}, h, \Gamma_{2}}^{\left(M_{p}\right)}(\psi) & \\
\quad \text { for } \varphi & \in L_{d_{1}}^{\left(M_{p}\right)}\left(\Gamma_{1}\right), \psi \in L_{d_{2}}^{\left(M_{p}\right)}\left(\Gamma_{2}\right) .
\end{aligned}
$$

By the Hahn-Banach theorem, (6) holds for $\varphi \in L_{d_{1}, h}^{\left(M_{p}\right)}\left(\Gamma_{1}\right), \psi \in L_{d_{2}, h}^{\left(M_{p}\right)}\left(\Gamma_{2}\right)$. Put $\zeta_{j}:=b_{j}+i \eta_{j}$ where $b_{j} \in \mathbb{R}^{n_{j}}$ with $a_{j}<b_{j}<d_{j}$ and $\eta_{j} \in \mathbb{R}^{n_{j}}(j=1,2)$. Since there exist $c_{j}, k_{j}\left(k_{j}:=\left(1+\left|b_{j}\right|\right) / h\right)$ such that

$$
q_{d_{j}, h, \Gamma_{j}}^{\left(M_{p}\right)}\left(e^{x_{j} \zeta_{j}}\right) \leq c_{j} \exp M\left(k_{j}\left(1+\left|\eta_{j}\right|\right)\right),
$$

where $\exp M\left(k_{j}\left(1+\left|\eta_{j}\right|\right)\right):=\prod_{i=1}^{n_{j}} \exp M\left(k_{j}\left(1+\left|\eta_{j}^{i}\right|\right)\right)$, the function $\Gamma_{j} \ni$ $x_{j} \mapsto e^{x_{j} \zeta_{j}}$ belongs to $L_{d_{j}, h}^{\left(M_{p}\right)}\left(\Gamma_{j}\right)(j=1,2)$. So we conclude from (6) that

$$
\left|A\left[e^{x_{1} \zeta_{1}}\right]\left[e^{x_{2} \zeta_{2}}\right]\right| \leq c_{A} c_{1} c_{2} \exp M\left(k_{1}\left(1+\left|\eta_{1}\right|\right)\right) \exp M\left(k_{2}\left(1+\left|\eta_{2}\right|\right)\right) .
$$

Let $\Phi \in L_{a_{1}, a_{2}}^{\left(M_{p}\right)}\left(\Gamma_{1} \times \Gamma_{2}\right)$. Then the Laplace transform $\mathcal{L} \Phi$ given by

$$
\mathcal{L} \Phi(\zeta):=\int_{\Gamma} \Phi(x) e^{-\zeta x} d x \quad \text { for } \operatorname{Re} \zeta>\mathrm{a}
$$

satisfies

$$
\left|\mathcal{L} \Phi\left(\zeta_{1}, \zeta_{2}\right)\right| \leq c q_{\left(a_{1}, a_{2}\right), 1, \Gamma_{1} \times \Gamma_{2}}^{\left(M_{p}\right)}(\Phi)=: c_{\Phi}<\infty .
$$

Put $Q\left(\zeta_{1}, \zeta_{2}\right):=Q_{1}\left(\zeta_{1}\right) Q_{2}\left(\zeta_{2}\right)$ with

$$
\begin{aligned}
Q_{j}\left(\zeta_{j}\right) & :=\left(\zeta_{j}-d_{j}-1\right)^{p_{0}+1} \prod_{p=p_{0}}^{\infty}\left(1-\frac{k_{j} \zeta_{j}}{m_{p}}\right) \\
& :=\prod_{i=1}^{n_{j}}\left(\zeta_{j}^{i}-d_{j}^{i}-1\right)^{p_{0}+1} \prod_{p=p_{0}}^{\infty}\left(1-\frac{k_{j} \zeta_{j}^{i}}{m_{p}}\right),
\end{aligned}
$$

where $m_{p}:=M_{p} / M_{p-1}, p_{0}$ is such that $m_{p}>2 k_{j}\left|b_{j}\right|+k_{j}$ and $\left|m_{p}-k_{j} \zeta_{j}\right| \geq$ $k_{j}\left|\zeta_{j}\right|$ for $p \geq p_{0}, j=1,2$. By the Hadamard factorization theorem (Propositions 4.5 and 4.6 in [2]), $Q$ is a symbol of class $\left(M_{p}\right)$ and it satisfies the inequality (see [3], Lemma 3)

$$
\frac{\exp M\left(k_{1}\left|\zeta_{1}\right|\right) \exp M\left(k_{2}\left|\zeta_{2}\right|\right)}{\left|Q\left(\zeta_{1}, \zeta_{2}\right)\right|} \leq \frac{K^{\prime}}{\left(1+\left|\eta_{1}\right|\right)^{2}\left(1+\left|\eta_{2}\right|\right)^{2}}
$$

with some $K^{\prime}<\infty$, where $\left(1+\left|\eta_{j}\right|\right)^{2}:=\prod_{i=1}^{n_{j}}\left(1+\left|\eta_{j}^{i}\right|\right)^{2}(j=1,2)$. 
Now we can write the mapping $\mathcal{I}_{M_{p}}^{-1}$ :

$$
\begin{aligned}
\mathcal{I}_{M_{p}}^{-1}(A)[\Phi]:=\left(\frac{1}{2 \pi i}\right)^{n_{1}+n_{2}} Q\left(D_{x_{1}}, D_{x_{2}}\right) \int_{b_{1}+i \mathbb{R}^{n_{1}}} & \int_{b_{2}+i \mathbb{R}^{n_{2}}} A\left[e^{x_{1} \zeta_{1}}\right]\left[e^{x_{2} \zeta_{2}}\right] \\
& \times \frac{\mathcal{L} \Phi\left(\zeta_{1}, \zeta_{2}\right)}{Q\left(\zeta_{1}, \zeta_{2}\right)} d \zeta_{1} d \zeta_{2} .
\end{aligned}
$$

From (7)-(9) we obtain

$$
\begin{aligned}
\left|A\left[e^{x_{1} \zeta_{1}}\right]\left[e^{x_{2} \zeta_{2}}\right] \frac{\mathcal{L} \Phi\left(\zeta_{1}, \zeta_{2}\right)}{Q\left(\zeta_{1}, \zeta_{2}\right)}\right| & \\
& \leq c_{A} c_{1} c_{2} c_{\Phi} \frac{\exp M\left(k_{1}\left(1+\left|\eta_{1}\right|\right)\right) \exp M\left(k_{2}\left(1+\left|\eta_{2}\right|\right)\right)}{\left|Q\left(\zeta_{1}, \zeta_{2}\right)\right|} \\
& \leq \frac{K}{\left(1+\left|\eta_{1}\right|\right)^{2}\left(1+\left|\eta_{2}\right|\right)^{2}}
\end{aligned}
$$

with some $K<\infty$. Therefore the integral in (10) is convergent (vector notation!).

Since the ultradifferential operator

$$
Q\left(D_{x_{1}}, D_{x_{2}}\right): L_{a}^{\left(M_{p}\right)}(\Gamma) \rightarrow L_{a}^{\left(M_{p}\right)}(\Gamma)
$$

is continuous (cf. Th. 2.12 in [2]), for $h>0$ sufficiently small we have

$$
\left|\mathcal{I}_{M_{p}}^{-1}(A)[\Phi]\right| \leq C c_{A} q_{\left(a_{1}, a_{2}\right), h, \Gamma_{1} \times \Gamma_{2}}^{\left(M_{p}\right)}(\Phi)
$$

with some $C<\infty$. Thus $\mathcal{I}_{M_{p}}^{-1}(A) \in L_{\left(\omega_{1}, \omega_{2}\right)}^{\prime\left(M_{p}\right)}\left(\Gamma_{1} \times \Gamma_{2}\right)$.

If a sequence $\left(A_{\nu}\right)_{\nu \in \mathbb{N}}$ is convergent to zero in $L_{\left(\omega_{1}\right)}^{\prime\left(M_{p}\right)}\left(\Gamma_{1}, L_{\left(\omega_{2}\right)}^{\prime\left(M_{p}\right)}\left(\Gamma_{2}\right)\right)$ then by Theorem 3 the sequence of the corresponding numbers $c_{A_{\nu}}$ in (6) converges to zero. Thus the sequence $\left(\mathcal{I}_{M_{p}}^{-1}\left(A_{\nu}\right)\right)_{\nu \in \mathbb{N}}$ is convergent to zero in $L_{\left(\omega_{1}, \omega_{2}\right)}^{\prime\left(M_{p}\right)}\left(\Gamma_{1} \times \Gamma_{2}\right)$ and we conclude that the operator $\mathcal{I}_{M_{p}}^{-1}$ is continuous.

Next we show that $\mathcal{I}_{M_{p}}^{-1}$ is the inverse mapping to $\mathcal{I}_{M_{p}}$. To this end we apply the operator $Q\left(D_{x}\right)$ to the inversion formula for the Laplace transformation (see [9]). For $\varphi \in L_{a}^{\left(M_{p}\right)}(\Gamma)$ we have

$$
\varphi(x)=Q\left(D_{x}\right)\left(\frac{1}{2 \pi i}\right)^{n} \int_{b+i \mathbb{R}^{n}} e^{x \zeta} \frac{\mathcal{L} \varphi(\zeta)}{Q(\zeta)} d \zeta, \quad \text { where } x \in \Gamma .
$$

From the above equality and Lemma 2 we derive that

$$
\mathcal{I}_{M_{p}}\left(\mathcal{I}_{M_{p}}^{-1}(A)\right)[\varphi][\psi]=\mathcal{I}_{M_{p}}^{-1}(A)[\varphi \otimes \psi]
$$

is equal to 


$$
\begin{aligned}
\left(\frac{1}{2 \pi i}\right)^{n_{1}+} & Q_{2}\left(D_{x_{1}}\right) Q_{2}\left(D_{x_{2}}\right) \int_{b_{1}+i \mathbb{R}^{n_{1}}} \int_{b_{2}+i \mathbb{R}^{n_{2}}} A\left[e^{x_{1} \zeta_{1}}\right]\left[e^{x_{2} \zeta_{2}}\right] \\
& \times \frac{\mathcal{L} \varphi\left(\zeta_{1}\right)}{Q_{1}\left(\zeta_{1}\right)} \frac{\mathcal{L} \psi\left(\zeta_{2}\right)}{Q_{2}\left(\zeta_{2}\right)} d \zeta_{1} d \zeta_{2} \\
= & \left(\frac{1}{2 \pi i}\right)^{n_{1}} Q_{1}\left(D_{x_{1}}\right) \int_{b_{1}+\mathbb{R}^{n_{1}}} \frac{\mathcal{L} \varphi\left(\zeta_{1}\right)}{Q_{1}\left(\zeta_{1}\right)} \\
& \times A\left[e^{x_{1} \zeta_{1}}\right]\left[\left(\frac{1}{2 \pi i}\right)^{n_{2}} Q_{2}\left(D_{x_{2}}\right) \int_{b_{2}+i \mathbb{R}^{n_{2}}} e^{x_{2} \zeta_{2}} \frac{\mathcal{L} \psi\left(\zeta_{2}\right)}{Q_{2}\left(\zeta_{2}\right)} d \zeta_{2}\right] d \zeta_{1} \\
= & \left(\frac{1}{2 \pi i}\right)^{n_{1}} Q_{1}\left(D_{x_{1}}\right) \int_{b_{1}+i \mathbb{R}^{n_{1}}} \frac{\mathcal{L} \varphi\left(\zeta_{1}\right)}{Q_{1}\left(\zeta_{1}\right)} A\left[e^{x_{1} \zeta_{1}}\right][\psi] d \zeta_{1} \\
= & A\left[\left(\frac{1}{2 \pi i}\right)^{n_{1}} Q_{1}\left(D_{x_{1}}\right) \int_{b_{1}+i \mathbb{R}^{n_{1}}} e^{x_{1} \zeta_{1}} \frac{\mathcal{L} \varphi\left(\zeta_{1}\right)}{Q_{1}\left(\zeta_{1}\right)} d \zeta_{1}\right][\psi] \\
= & A[\varphi][\psi] .
\end{aligned}
$$

Similarly we obtain

$$
\begin{aligned}
\mathcal{I}_{M_{p}}^{-1}\left(\mathcal{I}_{M_{p}}(\widetilde{A})\right)[\Phi] & \left(\frac{1}{2 \pi i}\right)^{n_{1}+n_{2}} Q\left(D_{x_{1}}, D_{x_{2}}\right) \\
& \times \int_{b_{1}+i \mathbb{R}^{n_{1}}} \int_{b_{2}+i \mathbb{R}^{n_{2}}} \frac{\mathcal{L} \Phi\left(\zeta_{1}, \zeta_{2}\right)}{Q\left(\zeta_{1}, \zeta_{2}\right)} \mathcal{I}_{M_{p}}(\widetilde{A})\left[e^{x_{1} \zeta_{1}}\right]\left[e^{x_{2} \zeta_{2}}\right] d \zeta_{1} d \zeta_{2} \\
= & \widetilde{A}\left[\left(\frac{1}{2 \pi i}\right)^{n_{1}+n_{2}} Q\left(D_{x_{1}}, D_{x_{2}}\right) \int_{b_{1}+i \mathbb{R}^{n_{1}}} \int_{b_{2}+i \mathbb{R}^{n_{2}}} e^{x_{1} \zeta_{1}+x_{2} \zeta_{2}} \frac{\mathcal{L} \Phi\left(\zeta_{1}, \zeta_{2}\right)}{Q\left(\zeta_{1}, \zeta_{2}\right)} d \zeta_{1} d \zeta_{2}\right] \\
= & \widetilde{A}[\Phi] .
\end{aligned}
$$

This completes the proof.

Acknowledgments. The author gratefully acknowledges many helpful suggestions of Grzegorz Łysik during the preparation of the paper.

\section{References}

[1] J. Horváth, Topological Vector Spaces and Distributions I, Addison-Wesley, Reading, MA, 1966.

[2] H. Komatsu, Ultradistributions, I. Structure theorems and a characterization, J. Fac. Sci. Univ. Tokyo 20 (1973), 25-105. 
[3] G. Eysik, Laplace ultradistributions on a half line and a strong quasi-analyticity principle, Ann. Polon. Math. 63 (1996), 13-33.

[4] R. Meise and A. Taylor, Linear extension operators for ultradifferentiable functions of Beurling type on compact sets, Amer. J. Math. 111 (1989), 309-337.

[5] L. Schwartz, Théorie des noyaux, in: Proc. Internat. Congress Math. Cambridge 1950, I, 220-230.

[6] Z. Szmydt, Fourier Transformation and Linear Differential Equations, PWN-Polish Sci. Publ., Warszawa, and Reidel, Dordrecht, 1977.

[7] Z. Szmydt and B. Ziemian, The Mellin Transformation and Fuchsian Type Partial Differential Equations, Kluwer, Dordrecht, 1992.

[8] - - - Topological imbedding of Laplace distributions in Laplace hyperfunctions, Dissertationes Math. 376 (1998).

[9] D. V. Widder, The Laplace Transform, Princeton Univ. Press, Princeton NJ, 1946.

Institute of Mathematics

Polish Academy of Sciences

P.O. Box 137

Śniadeckich 8

00-950 Warszawa, Poland

E-mail: slawek@impan.gov.pl

Reçu par la Rédaction le 19.11.1998

Révisé le 26.2.2001 